\title{
Further studies of the production of isolated photons at HERA
}

\section{P. J. Bussey}

School of Physics and Astronomy

University of Glasgow

Glasgow, United Kingdom, G12 8QQ

E-mail: peter.bussey@glasgow.ac.uk

for the ZEUS Collaboration

The ZEUS detector at HERA has been used to measure the production of isolated photons in two types of process: deep inelastic scattering (DIS) and diffractive photoproduction. The DIS results are presented as functions of two- and multi-particle final-state quantities, extending an earlier publication. Comparison is made with two NLO-based theoretical calculations. In diffraction, cross sections are evaluated in terms of a number of variables, differentiating between direct and resolved photon and pomeron processes. Evidence is found for the existence of a direct pomeron process in this reaction. The shapes of all the distributions are consistent with predictions from the RAPGAP Monte Carlo simulation.

The 39th International Conference on High Energy Physics (ICHEP2018)

4-11 July, 2018

Seoul, Korea 
The production of prompt photons in hadronic reactions can provide a viewpoint on the scattering process which is not strongly dependent on the modelling of jet fragmentation. Here, we report on two recent analyses by the ZEUS collaboration using data obtained with the HERA electron-proton collider. "Electron" will refer to both $e^{-}$and $e^{+}$incoming leptons. The ZEUS barrel electromagnetic calorimeter is sufficiently fine-grained to enable a separation of photon signals from the broader signals that arise from the decay of neutral mesons. The separation is performed statistically in each bin of each measured variable by fitting simulated signals due to a photon and to background arising from neutral meson decay. To minimise the contribution from photons produced in jets, and to reduce the background, the photons were required to be isolated, such that in any jet-like object containing the outgoing photon, at least $90 \%$ of the energy must belong to the photon signal.

The first analysis presented here is of events that contain a high-energy outgoing photon and a jet in DIS collisions, extending an earlier ZEUS analysis [1]. A number of variables are measured based on information from two or more of the scattered objects in the event. These are the variable $x_{\gamma}^{\text {meas }}$, which gives information about the fraction of the exchanged photon energy-momentum that emerges in the photon-jet system, a corresponding variable for the incoming proton, and differences in pzeudorapidity and azimuth between outgoing particles. The cross-sections are obtained by correcting for acceptance by means of a PYTHIA/HERACLES-based simulation.

Results are shown in fig. 1. Comparisons are made to two theoretical calculations. It can be seen that the AFG calculation [3], while not perfect, provides a reasonably good description of the data in all the variables that are plotted. The BLZ calculation [4] is satisfactory for many of the variables, but less good in the $x_{\gamma}^{\text {meas }}$ and $\Delta \phi$ distributions.

Diffractive hadronic interactions involve the exchange of a colour-neutral object known as the "Pomeron". Diffractive scattering off protons may be initiated by a second incoming hadron, or by a real or virtual photon. The diffractive process is characterised by a forward nucleon, followed by a "gap" in rapidity in which little or no energetic scattering is found until the central region of the process where the hard final state is detected and measured. ZEUS has measured diffractive photoproduction at HERA in which a hard isolated "prompt" photon may be accompanied by a jet [5]. In such processes, the outgoing photon must be radiated from a charged partonic object, namely a quark, and therefore demonstrates the presence of a quark content in the Pomeron or of scattering in which both the Pomeron and incident photon couple to quarks.

When a proton, with energy $E_{p}$, radiates a Pomeron or equivalent, the fraction of the proton energy taken by the radiated Pomeron is given to a good approximation by the variable $x_{\mathbb{P}}^{\text {meas }}=$ $\left(E^{\text {all }}+p_{Z}^{\text {all }}\right) / 2 E_{p}$, where the suffix "all" refers to all final-state particles, or detector-measured objects, apart from the forward proton and its possible dissociation products. In "direct" photoproduction processes the entire energy of the incoming photon is absorbed by the target, while in "resolved" processes the incoming photon's hadronic structure provides a quark or gluon that interacts with a parton from the target. These processes may be partially distinguished by means of the variable $x_{\gamma}^{\text {meas }}=\left(E^{\gamma}+E^{\text {jet }}-p_{Z}^{\gamma}-p_{Z}^{\text {jet }}\right) /\left(E^{\text {all }}-p_{Z}^{\text {all }}\right)$, which measures the fraction of the incoming photon energy that is given to the photon and the jet. The quantities $E^{\gamma}$ and $E^{\text {jet }}$ denote the energies of the photon and the jet, respectively; $p_{Z}$ denotes the corresponding longitudinal momentum. The presence of direct processes generates a prominent peak in the cross section at high 

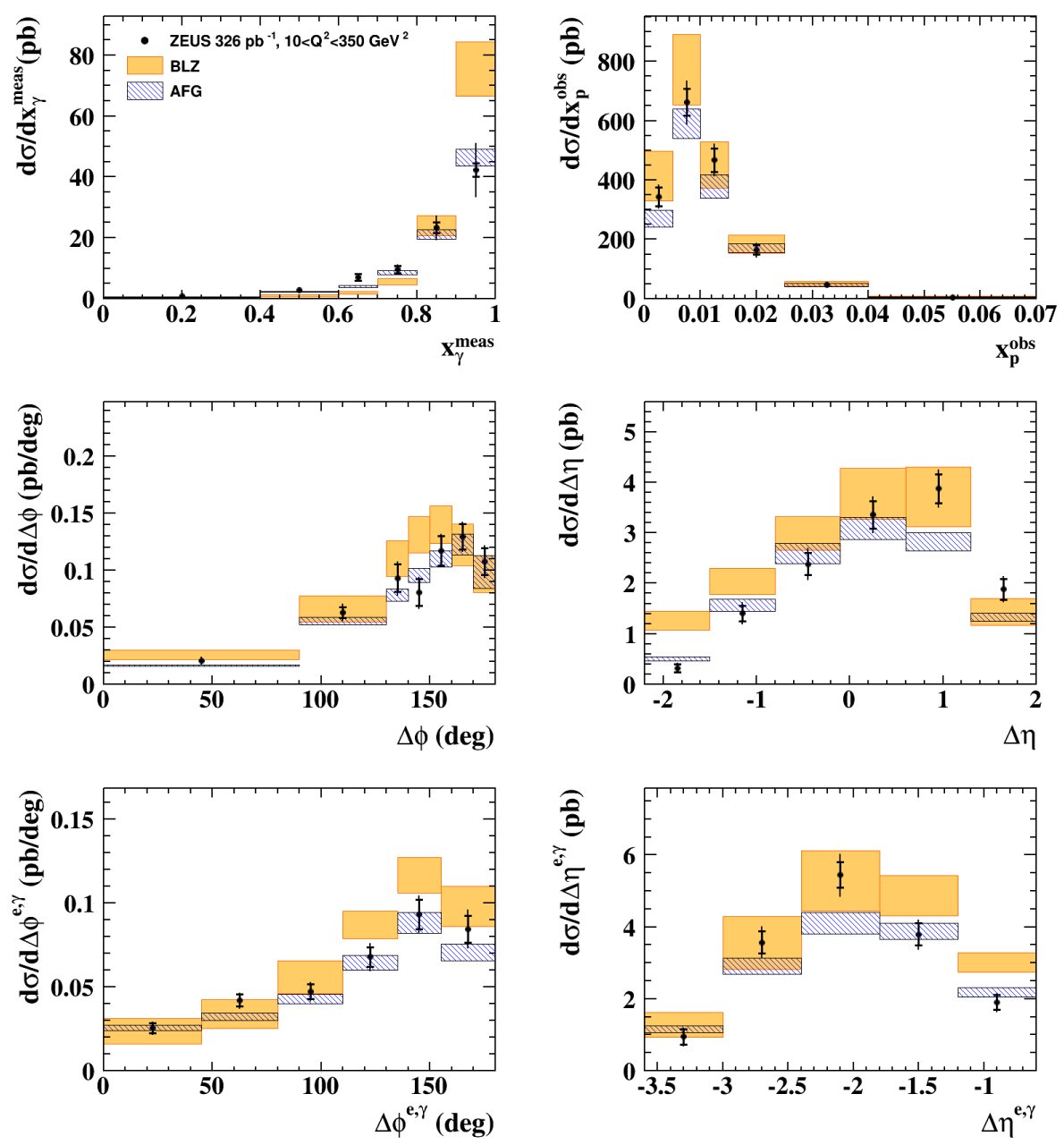

Figure 1: Examples of measured variables in the production of prompt photons with a jet in deep inelastic scattering, in comparison with NLO theoretical predictions. Thick error bars are statistical and thin are statistical combined with systematic.

$x_{\gamma}^{\text {meas }}$ values. Similarly, direct and resolved Pomeron processes may be defined. The fraction of the Pomeron energy that is taken by the outgoing photon and jet is given to a good approximation by: $z_{\mathbb{P}}^{\text {meas }}=\left(E^{\gamma}+E^{\text {jet }}+p_{Z}^{\gamma}+p_{Z}^{\text {jet }}\right) /\left(E^{\text {all }}+p_{Z}^{\text {all }}\right)$.

The measurements are based on two data samples corresponding to integrated luminosities of 82 and $374 \mathrm{pb}^{-1}$, taken during the years 1998-2000 (HERA-I) and 2004-2007 (HERA-II). Monte Carlo (MC) event samples were prepared using RAPGAP 3.2 to generate the diffractive process and PYTHIA 6.416 to generate prompt-photon photoproduction processes for background calculations.

Events were finally selected with the following kinematic conditions. Each event was required to contain a photon candidate with a reconstructed transverse energy, $E_{T}^{\gamma}$, in the range $5<E_{T}^{\gamma}<15 \mathrm{GeV}$ and with pseudorapidity, $\eta^{\gamma}$, in the range $-0.7<\eta^{\gamma}<0.9$. The hadronic jet, when used, was required to have $E_{T}^{\text {jet }}$ between 4 and $35 \mathrm{GeV}$ and to lie within the pseudorapidity range $-1.5<\eta^{\text {jet }}<1.8$. To select diffractive events two further conditions were applied: (i) the 
(a)

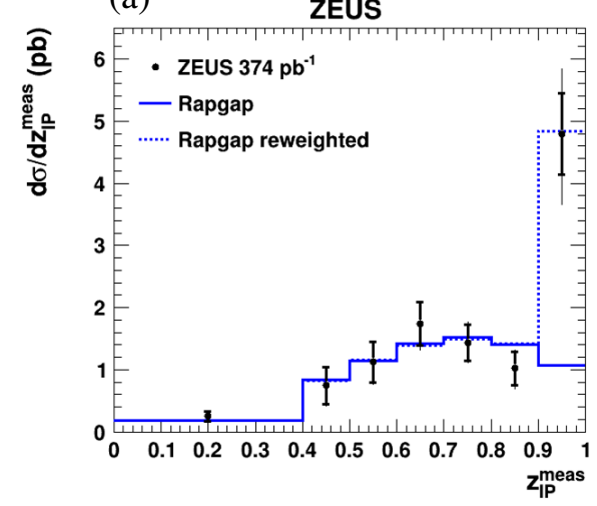

(b)

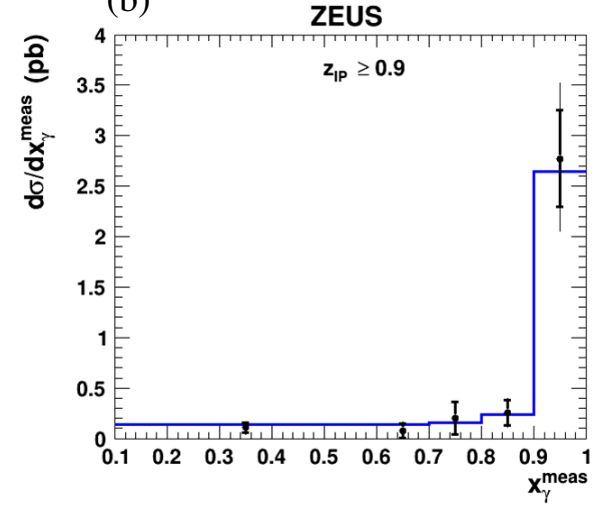

Figure 2: Differential cross sections for events containing an isolated photon accompanied by a jet: (a) as a function of $z_{\mathbb{P}}^{\text {meas }}$. The solid RAPGAP histogram is normalised to the region $z_{\mathbb{P}}^{\text {meas }}<0.9$, while the dashed histogram includes a reweighting factor and is normalised to the entire data distribution. (b) as a function of $x_{\gamma}^{\text {meas }}$ for events with $z_{\mathbb{P}}^{\text {meas }}>0.9$ at the hadron level.

maximum pseudorapdity for EFO's (detected tracks or calorimeter signals) with energy above 0.4 $\mathrm{GeV}$ was less than 2.5, and (ii) $x_{\mathbb{P}}^{\text {meas }}<0.03$. A serious potential background was of Bethe-Heitler events containing a final-state high- $E_{T}$ photon and electron. These and DIS events were efficiently removed by rejecting events with an identified electron and fewer than six EFO's in the detector.

The results are potentially affected by proton-dissociation processes, in which the products of the proton dissociation pass undetected inside the central aperture of the Forward Calorimeter. These were not modelled in the version of RAPGAP that was used and were estimated to be up to $40 \%$ for the HERA-II data and up to $16 \%$ for the HERA-I data. The HERA-II data were used to give the best estimation of the shapes of the cross-section distributions, which were assumed not to depend on the presence of proton dissociation. The HERA-I data were were used to evaluate the total cross section within the selected parameter range. This was found to be $0.68 \pm 0.14_{-0.07}^{+0.06} \mathrm{pb}$, to be compared to the RAPGAP prediction of $0.68 \mathrm{pb}$ with no proton dissociation and no resolvedsuppression factor.

Figure 2(a) shows the differential cross section for $z_{\mathbb{P}}^{\text {meas }}$. At the upper end of the distribution, a peak is seen which is not described by RAPGAP and which gives evidence for the presence of direct-Pomeron processes. Figure 2(b) shows the $x_{\gamma}^{\text {meas }}$ cross section for events with $z_{\mathbb{P}}^{\text {meas }}>0.9$ and shows that these events are dominated by a direct-photon component. It is found that RAPGAP describes well the shapes of all the measured variables, both for $z_{\mathbb{P}}^{\text {meas }}<0.9$ and for $z_{\mathbb{P}}^{\text {meas }}>0.9$.

\section{References}

[1] ZEUS Collaboration, H. Abramowicz et al., Phys. Lett. B 175 (2012) 88.

[2] ZEUS Collaboration, H. Abramowicz et al., JHEP 1801 (2018) 032.

[3] P. Aurenche, M. Fontannaz and J-Ph. Guillet, Eur. Phys. J. C44 (2005) 395.

[4] S. Baranov, A. Lipatov and N. Zotov, Phys. Rev. D81 (2010) 094034.

[5] ZEUS Collaboration, H. Abramowicz et al., Phys. Rev. D06 (2017) 032006. 